\title{
Image Registration Based On a Novel Enhanced Scale Invariant Geometrical Features
}

\author{
Ehsan Hossein KalatehJari, Mohammad Mehdi Hosseini, and Abdorreza Alavi Gharahbagh
}

\begin{abstract}
Image registration can find similarities between one image and another one from the same scene taken by different angles. Registration is widely used in robot localization, remote sensing, medical imaging and etc. In this paper, a new method based on the image geometrical features has introduced by using two important characteristics of image scale and image rotation. Feature points of two images by SIFT algorithm has extracted. Then, an initial matching is estimated based on descriptor matrix of SIFT features with nearest neighbor (NN) criterion. The novel geometrical method is used for discarding incorrect matched points. Finally, the correct image is recognized by the number of matched points.
\end{abstract}

Index Terms-Nearest neighbor, sift, geometrical transform, image registration.

\section{INTRODUCTION}

Image registration is the process for aligning one image with another one from the same scene taken at different times or from different angles or by different sensors. There are a few different image registration methods that each of them is used for a specific application. However, general image registration methods according to brown [1] is considered as a combination of four distinctive parts: Feature Space that provides information about image adjusting, Search space or conversion model that is a Group of transfer functions with ability of matching two images, Likeness criteria that is used for relative similarity measurement between feature pairs and finally search method that determines how to choose the next transforms of feature space for achieving an optimum transform.

Image features are special characteristics of image that can be used for finding correspondence similarity between two images. Most desirable characteristics are feature points because their coordinates can be used for finding the corresponding transfer function that makes the image registration. However, in some images it is not possible to determine feature points. In these cases, high Lines or areas in the image can be used as image features. There are various methods for extracting corner and lines such as Moravec operator [2], the method of Kitchen and Rosenfeld [3], KLT [4] and the Harris algorithm [5] Hough transform [6], Minimum Squares Method [7], Segmentation methods, threshold, etc.

An important aspect of image registration methods is

Manuscript received July17; revised August 30, 2012.

The authors are with the department of electrical and computer engineering, Islamic azad university, shahrood branch, shahrood, Iran. (e-mail: ehsankl@yahoo.com; Hoseini_mm@yahoo.com; dramalavi_gharah@yahoo.com) transforming model and defining geometrical mismatches between images and finding model and applying it to the image. For this case, a local or global transform model can be used. Global transform model is applied on the total image such as projective, affine, rigid, and curved while parameters of local transform such as piecewise interpolation, Tine-Plate spline and continuum mechanic change in different parts.

Another important issue that must be considered is similarity criteria that are categorized into two general categories: 1. criteria based on intensity 2. Criteria based on characteristics. In addition, several hybrid approaches have been suggested that provides advantage of both methods.

Scale change is one of the transfer function parameters that make difficulties in image matching methods, especially in feature extraction. For a large scale factor, area of overlap shrinks and a few points can't reliably represent in image. Therefore the selected method should be enough robust against high scale change and can spot a large number of feature points especially when image texture changes rapidly such as outdoor images. The possibility of extract correct matches can be improved by increasing the number of points. In this paper, a new enhanced feature matching that is robust against scale changes is proposed.

\section{FEATURE POINT EXTRACTING}

In this paper, only feature points are used for image registration. These features must be invariant against image scaling and rotation. Therefore SIFT are the best algorithm that have been introduced for feature point extracting. In this section we briefly review scale invariant feature point's detector, descriptor and the criteria used to measure matching cost.

\section{A. Sift Feature}

Lowe [8] developed SIFT (Scale Invariant Feature Transform) for Image feature generation in object recognition applications. These features are stable against scaling, rotation, and partly illumination changes. For all image points, the value of corniness evaluation function (CEF) is computed in many successive resolutions or scales $\left(\delta_{s}\right)$. The discrete values of scale are distributed exponentially between the inner and outer limits $\delta_{s}=\delta_{0} r^{n}$.

The CEF in 3D space (x, y, $\boldsymbol{\delta}$ ), has a local extremum. Its value is more than a certain threshold and named scale invariant feature point. The detector allocates to each feature point a spatial location $X_{p}=\left(x_{p}, y_{p}\right)$ and a characteristic 
scale $\delta_{p}$. Also, some descriptors need a standard orientation $\theta_{\text {std }}$ to become rotation invariant. This orientation is usually the dominant local gradient orientation in a support region around feature point. The local image gradients are measured at the selected scale in the region around each keypoint. Supported region is rotated such that assigned orientation to feature point lay on a canonical direction. Gradient direction at each sample point in support region is weighted by gradient magnitude. Then, supported region is divided to 16 sub-regions $(4 \times 4)$. An orientation histogram with 8 bins is created by weighted gradient orientations of each sub region. Therefore, a vector with $16 \times 8=128$ components is obtained that is descriptor matrix of each key point.

To compensate inexactness on assigned characteristic scale a Gaussian weighting function is used to reduce weight of gradients that are far from the center of the support region. These are transformed into a representation that allows for significant levels of local shape distortion and change in illumination. All future operations are performed on image data that has been transformed relative to the assigned orientation, scale, and location for each feature, thereby providing invariance to these transformations. Fig 1 shows an image with extracted SIFT features that distributed all over the image.

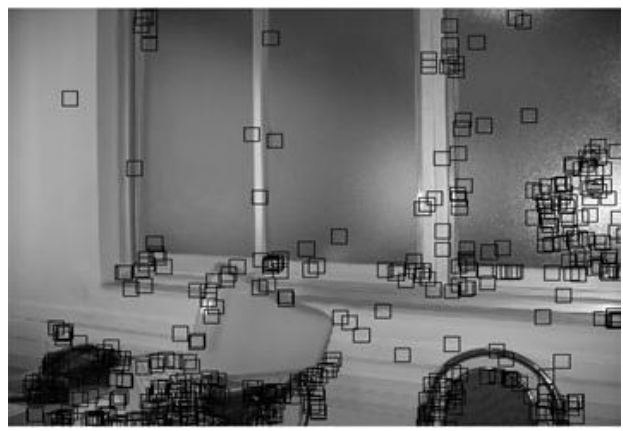

Fig. 1. Feature points for a typical image

\section{INITIAL MATCHING}

In this step description matrix is used for finding feature point similarity between two images. The nearest neighbor (NN) method is used for finding Key point matching. The Euclidean distance for the invariant descriptor vector is described in the following equation:

$$
\operatorname{DIS}(j)=\sqrt{\sum_{i=1}^{128}\left(q_{i}-q_{i}^{\prime}\right)^{2}}
$$

Which $q_{i}$ is related to the description matrix of tested feature, $q_{i}^{\prime}$ is related to features of reference image and $\mathrm{J}$ indicates which feature has been compared. A global threshold on closest distance doesn't have a good performance therefore, ratio between the distances of closest neighbor with respect to distance of the second-closest neighbor has proposed as a measure to reject outliers, by applying a threshold on this ratio significant number of false matches are rejected. At the same time, many correct matches are lost and many incorrect matches remain. In this step a local photometrical criterion in combination with a global geometrical method is used for better results.

\section{REJECTING OUTLIER KEYPOINTS}

As mentioned before a local research was used for finding primary matching and a global research must be applied for discarding incorrect matches. Criteria of scale change and rotation are one of the most important aspects of image transforms like affine and projective transform that are geometrical features of an image. In this paper, projective transform is used for computing image conversions; it is predictable that these changes be applied over the entire image components therefore, scale and rotation changes are applied on most existing elements of image. If we consider the lines between feature points as elements of an image, there are related lines corresponding to matching points in another image that rotation and scale changes between two related lines can be considered as common features. The author's goal is to use these two features for classification correct feature point matching and incorrect matching points. For clarification purpose, each feature has explained separately. Fig. 2 shows the steps of proposed algorithm.

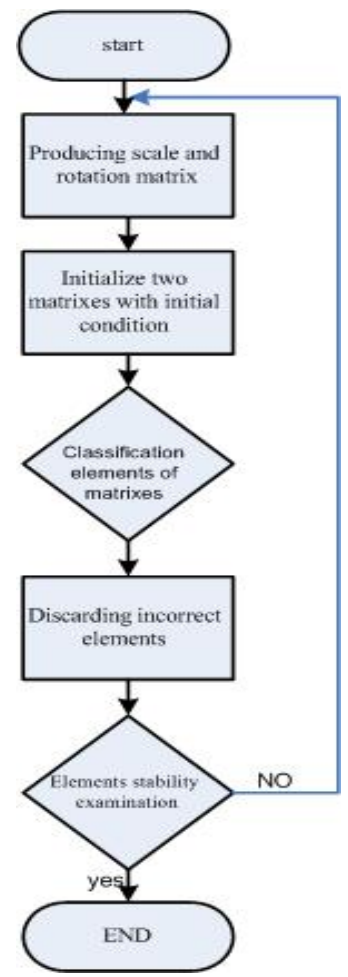

Fig. 2. Flowchart of steps of proposed algorithm

\section{A. Image Scale Changes as geometrical feature}

Suppose $p=\left\{p_{1}, p_{2}, \ldots ., p_{n}\right\}$ be feature points extracted from training image and $q=\left\{q_{1}, q_{2}, \ldots ., q_{n}\right\}$ is

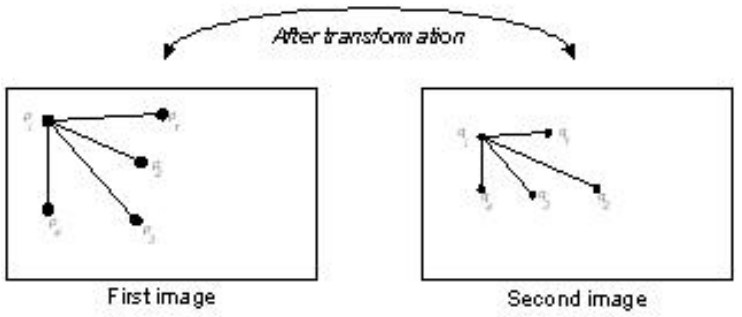

Fig. 3 .Scale change between two images 
Corresponding feature points in the given image.

Step1. Let $S_{i, j}$ be similarity measurement between

$p_{i}$ and $q_{i}$, the Distance between these points and other key points is provided as shown in Fig 3. Various scale transforms are applied on the connecting lines between feature points and so the distance ratio of target lines and reference lines can be used as shown in equation 2 .

$$
S C A(i, j)=\frac{\square p_{i}-p_{j} \square}{\square q_{i}-q_{j} \square}
$$

Suppose there are $\mathrm{M}$ pairs $\left\{(p, q)_{k}\right\}$ for every point. Therefore, $\mathrm{M}-1$ neighbor points that can produce $\mathrm{M}-1$ related distinctive lines in both images can be found.

Step2. The scoring matrix SM as illustrated in Fig 4 is considered. i'th row of this matrix belongs to the pair labeled with $\left(p_{i}, q_{i}\right)$. Geometrical distance of other pairs can be estimated with respect to this pair. jth column of this row consist of pairs that satisfied equation 3 , with a tolerance.

$$
\begin{aligned}
& S M(i, j)=[(k \mid\|S C A(i, j)-S C A(i, k)\|<\delta, \\
& 1 \leq k \leq M, k \neq j]
\end{aligned}
$$

When there is no line between $\mathrm{i}=\mathrm{j}$, diagonal elements of SM matrix are equal to zero.

Step3. After filling all elements of matrix, the elements that their members are more than a specific threshold are considered and other pairs with related low element members are discarded because remaining elements refer to lines between two points. There are two choices for reference point in a line that leads to same scale changes in both of them; therefore correct matched point elements of matrixes must be diagonal. This characteristic can be used.

for discarding other points in correct matched points. The third characteristic of matrix is that the remaining elements must have the same members, so:

$$
\begin{aligned}
& A=\left\{j \mid j \subseteq\left[\operatorname{scle}\left(i, k_{1}\right) \cap \operatorname{scle}\left(i, k_{2}\right) \cap \ldots . .\right],\right. \\
& 1 \leq k \leq M, k \neq j\}
\end{aligned}
$$

Which, $A$ refers to a set of correct matching points.

\section{B. Image Rotation as geometrical feature}

Another important parameter in image transform functions is rotation angle. The following condition has considered:

$$
\square \theta\left(p_{1}\right)_{2}-\square \theta\left(q_{1}\right)_{2}=R O T(1,2)
$$

where $\theta\left(p_{1}\right)_{2}$ is angle of $\overrightarrow{p_{1} p_{2}}$ and $\theta^{\prime}\left(q_{1}\right)_{2}$ refers to angle of $\overrightarrow{q_{1} q_{2}}$. A new matrix is made and the same method for discarding incorrect matched points is used. Finally, there are two different matrixes that can be use for finding correct matched points of common elements between them. An iterative manner for more accuracy is used until stabilization of remaining points. At this stage, there isn't any reduction in number of remaining feature points (Fig. 4).

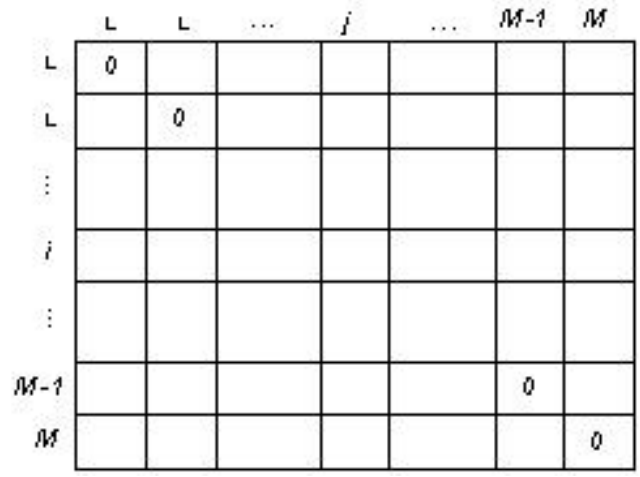

Fig. 4. SM matrix consist of similarity measurement

To confirm the correct matches the criterion in [10 11] has been used. A match is correct if the error in relative location is less than 3 pixels and the error in image area covered by two corresponding point neighbors is less than $30 \%$ of the region union.

\section{EXPERIMENT RESULT}

Combination of indoor and outdoor image pairs with difference scale changes and rotation angles, outdoor images and indoor images are selected from Google-earth and an office respectively. The scale factor in outdoor images is in the range [1 6] and in the indoor images is in the range [0.4 $3.5]$ the rotation angles are in the range [-120o 120o].

As mentioned in section 2, to prevent losing correct matches restricted threshold on matching cost is not applied. Fig 5 shows the ratio between correct matches to all detected matches. According to the results, $\mathrm{P}_{\text {th }}=0.6$ for achieving best result has been selected. Therefore, significant number of false matches is remaining. To discard these points, a robust shape matching approach is used and results are compared with two common method that are widely used for this goal, Hough transform and LMSE criterion. After removing false matching points in each method, instead of selecting image with maximum matched points, image with respect to ratio of next maximum to maximum matched point number is selected. Fig. 6 shows candidate images after initial investigation by using proposed threshold.

In LMSE (Least Median Square Error) criterion, after finding the projective transform between training image and given image, this transform function is applied to the feature points of training image and then coordinate error is estimated between transformed points and existing points in the given image. LMSE criterion is used for choosing correct matches.

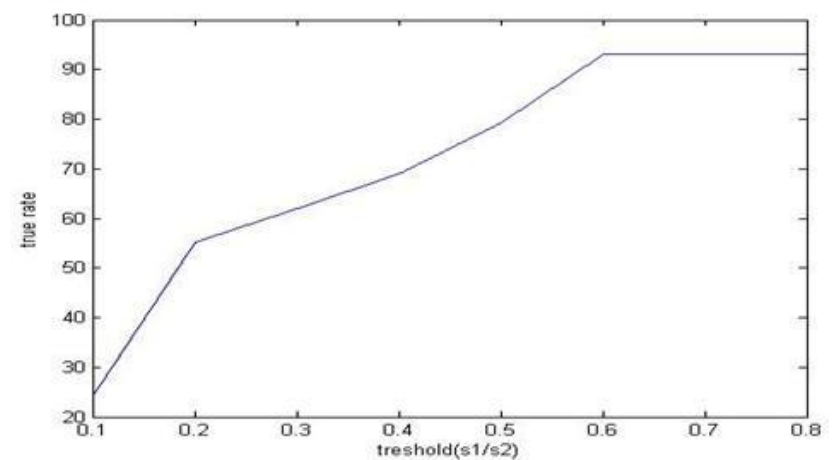

Fig. 5. True matching cost related to ratio of distance (closest/next closest) 


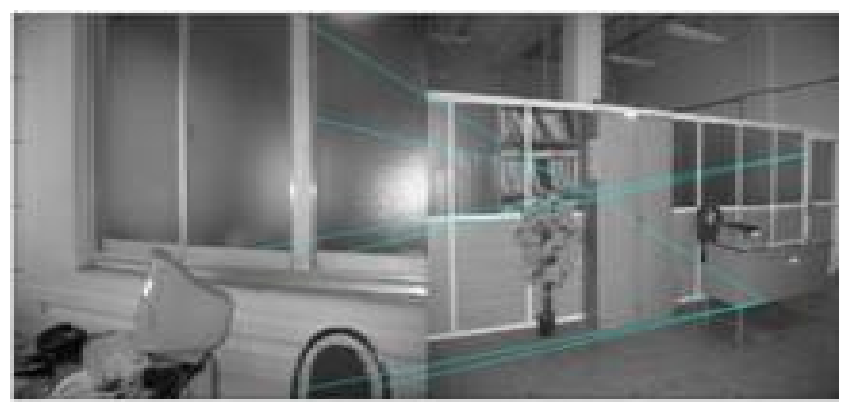

(a) First candidate image

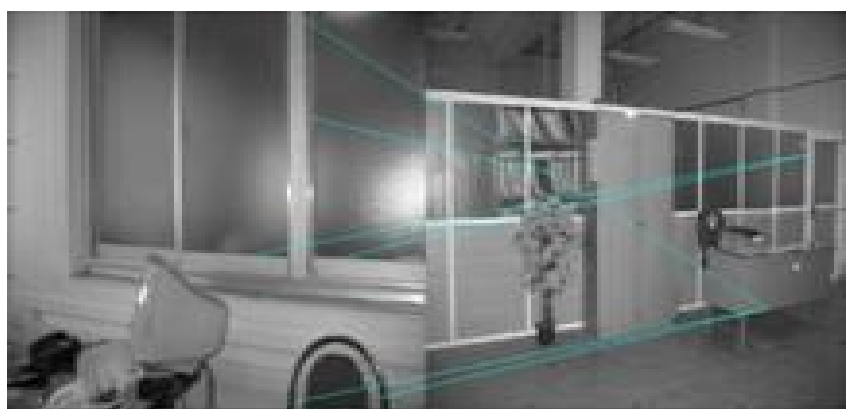

(b) Second candidate image

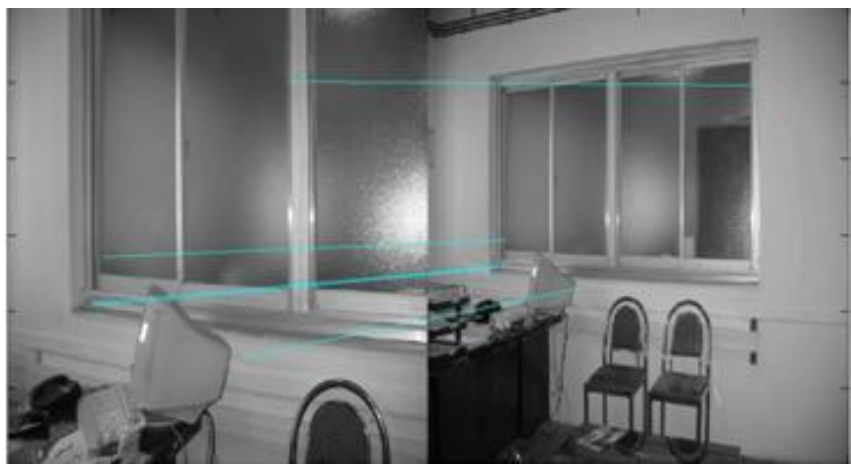

(c) Third candidate image

Fig. 6. Candidate images after initial investigation by using proposed threshold

David Lowe [8] used Hough transform for clustering feature points and removing false matching. After grouping feature points, each group is assigned to an object and therefore instead of number of points, number of objects is used. Finally, the results of using novel geometrical method, projective transform and Hough transform are shown in Fig 7, 8 that are the results of $\mathrm{P}_{\text {Correct }}$ and $\mathrm{P}_{\text {False-positive with respect to }}$ different ratio. In this method, false matched points are removed according to geometrical features of images. Fig 9 shows remaining feature points and image after applying proposed algorithm.

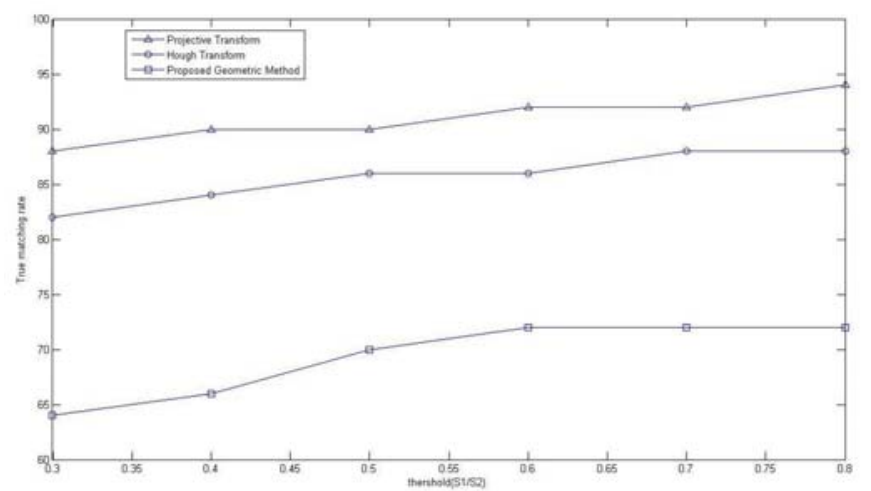

Fig. 7. True matching for projective, Hough and novel transform

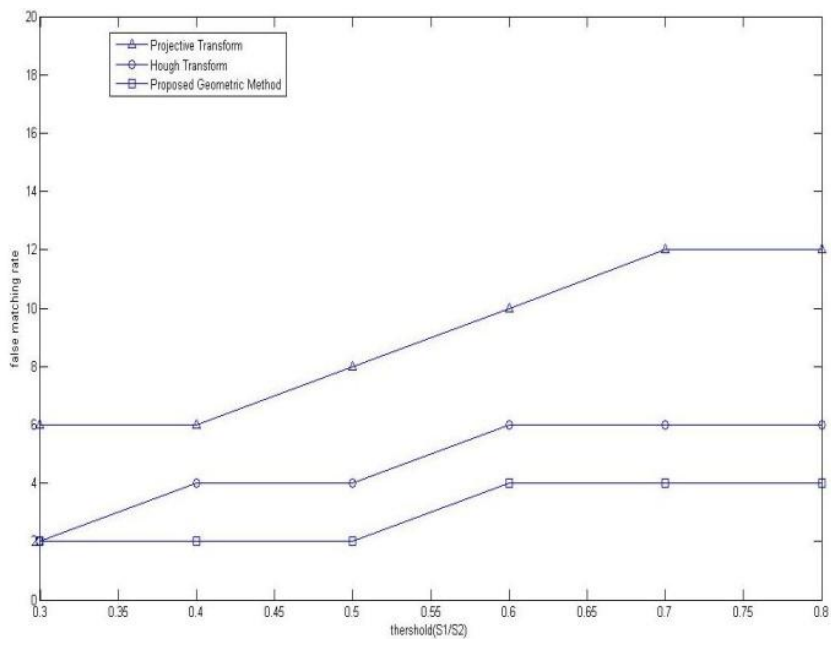

Fig. 8. False matching for projective, Hough and novel transform

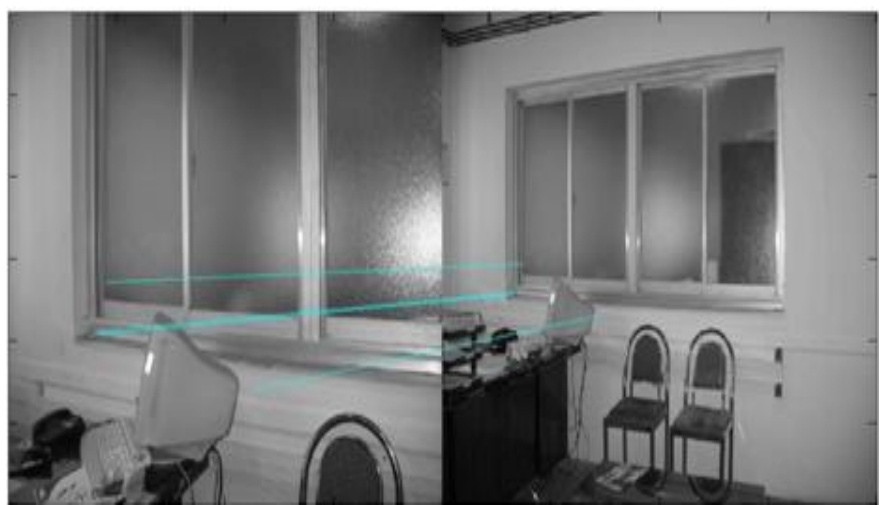

Fig. 9. Final output matching points and image

\section{CONCLUSiOnS}

LMSE is not a good method for removing false matches, because if the number of incorrect matches in an image is more than number of correct matches, this method cannot be used or when some of feature points are matched to a single point, the found error is low but the matching point is an incorrect point. Hough transform method has better results comparing to the first method but when matching points doesn't related to a same object or are far from each others, this method can't find any object and therefore the result is incorrect. The suggested method in this study has less faults and more efficiency but in crowded images, may show some weakness. In correct matches remaining and correct image doesn't be detected. If these features can be grouped and this criterion applied according to features of each group, the results can be optimizes. This method can be used for clustering features and finding object in the image or object tracking projects.

\section{REFERENCES}

[1] L. G. Brown, "A survey of image registration techniques," ACM Computing Surveys; 24(4), pp.325-376, Dec.1992.

[2] H. Moravec, "Visual mapping by a robot rover," In Proceedings of the 6th International Joint Conference on Artificial Intelligence, Tokyo, Japan, pp.598-600, 1979

[3] L. Kitchen and A. Rosenfeld, "Gray Level Corner Detection," Pattern Recognition Letters, 1: pp.95-102, 1989.

[4] J. Shi and C. Tomasi, “Good Features to Track,” IEEE Conference on Computer Vision and Pattern Recognition (CVPR94), pp.8-14, 1994. 
[5] C. G. Harris and M. Stephens, "A Combined Corner and Edge Detector," Proceeding of the 4th Alvey Vision Conference; pp. 147-151, 1988.

[6] Illingworth, J. and J. Kittler, "A survey of the Hough transform, Computer Vision, Graphics, and Image processing,” 44: pp.87-116, 1988.

[7] Rivlin, T. J. , "An Introduction to the Approximation of Functions," Blaisdell Publishing ; pp.48-65, 1969.

[8] Lowe, David G., “ Distinctive Image Features from Scale-Invariant Keypoints," International Journal of Computer Vision, 60[2]: pp. 91-110. Nov. 2004.

[9] K. Mikolajczyk, C. Schmid, "A performance evaluation of local descriptors,” CVPR, [2]: pp.257-263, June 2003.

[10] K. Mikolajczyk and C. Schmid, "An affine invariant interest point detector,” ECCV, pp.128-142, 2002.

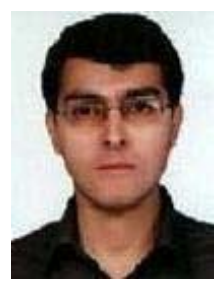

E. H. kalatehJari received the electrical Engineer degree from Shahrood University of Technology, Shahrood, Iran and the M.Sc. degree from the Islamic Azad University, Science and Research Tehran branch, Tehran, Iran. He works at the Islamic Azad University, shahrood branch, shahrood, Iran.

His research interests Include Image Processing and object Recognition.

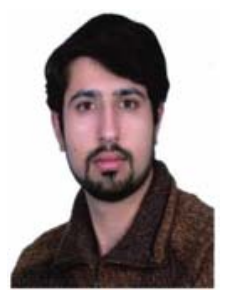

M. M. Hosseini received the electrical Engineer degree from Shahrood University of Technology, Shahrood, Iran and the M.Sc. degree from the Islamic Azad University, South Tehran branch, Tehran, Iran. He works at the Islamic Azad University, shahrood branch, shahrood, Iran.

His research interests Include Image Processing and pattern Recognition.

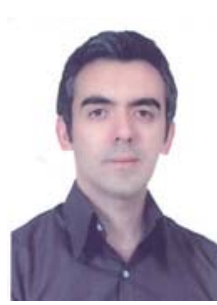

A. A. Gharahbagh received the communication Engineer degree from the ferdowsi University of mashhad, mashhad, Iran and the M.Sc. degree from the Urmia University, Urmia , Iran. He is currently a $\mathrm{PhD}$ student at Semnan university, Semnan, Iran.

His research activities focus on the artificial intelligence, optimization, Genetic Algorithm and image processing. He works at the Islamic Azad University, shahrood branch, shahrood, Iran. 